\title{
CORONAVIRUS MHV-A59 CAUSES UPREGULATION OF INTERFERON- $\beta$ RNA IN PRIMARY GLIAL CELL CULTURES
}

\author{
Q. Wang, J. A. Haluskey, and E. Lavi \\ Division of Neuropathology \\ Department of Pathology and Laboratory Medicine \\ University of Pennsylvania, School of Medicine \\ Philadelphiam, Pennsylvania 19104
}

\section{ABSTRACT}

Infection of mice with coronavirus mouse hepatitis virus strain MHV-A59 causes focal acute encephalitis, hepatitis and chronic demyelinating disease. To investigate host interferon (IFN) response to viral infection within the brain, RNA was extracted from A59-or MHV-2- infected and mock-infected primary astrocyte cultures from newborn mice, RT-PCR amplified RNA with primers specific for the various IFNs, transferred to nylon membranes and hybridized with IFN specific digoxigenin-labeled probes. Infection of primary astrocyte cultures from newborn mice with either A59 or MHV-2 caused upregulation of IFN- $\beta$ RNA, but not IFN- $\gamma$ or IFN- $\alpha$. Thus, brain astrocytes are capable of producing a local IFN- $\beta$ response upon infection with MHV. The response of the other IFNs following MHV infection may be derived from inflammatory cells.

\section{INTRODUCTION}

The pathologic hallmarks the chronic experimental disease induced by MHV infection in mice are similar to the human multiple sclerosis MS (Lavi et al., 1984a; Lavi et al., 1984b; Lavi et al., 1987a; Lavi and Weiss, 1989). As in MS, MHV-induced demyelination is believed to be immune-mediated (Wang et al., 1990). We initiated our investigation of primary glial cultures because astrocytes are considered as immune modulating cells in the CNS and one of the primary cell targets in MHV infection (Lavi et al., 1987a). We have previously shown that MHV infection causes upregulation of MHC class I expression in astrocytes and that astrocytes are the source of the MHC class I inducing factor 
(Lavi et al., 1987b; Lavi et al., 1989; Suzumura et al., 1988; Suzumura et al., 1986). Interferon response is an important antiviral, non-specific, host defense mechanisms. IFN- $\beta$ is an antiviral and immune modulating factor, an $\mathrm{MHC}$ inducer, and is known to be secreted by astrocytes among other cells. IFN- $\beta$ is used as one of the few treatment modalities in chronic demyelinating diseases such as MS although the mechanism of action of IFN- $\beta$ in MS is not clear. In the present investigation we asked whether MHV infection causes upregulation of IFN- $\beta$ in astrocytes. If so, what role may IFN- $\beta$ play in MHV infection and CNS disease. We used two strains of MHV for this investigation. While MHV-A59 causes focal acute encephalitis, hepatitis and chronic demyelinating disease, (MHV-2), a closely related strain, causes acute meningitis and hepatitis without significant involvement of brain parenchyma. The study of the differences between these two strains may give us some insights into the mechanism of MHV-induced CNS disease.

\section{METHODS AND MATERIALS}

\subsection{Preparation of Astrocyte Cultures}

Late-term pregnant, virus-free, C57BL/6 mice were purchased from Jackson Laboratories, Bar Harbor Maine. Astrocyte-enriched glial cell cultures from 2-3-day-old neonatal mice were estabalished by the modification of the method of MaCarthy as described previously (McCarthy and de Vellis, 1980). Briefly, brains were removed and passed through 19G, $21 \mathrm{G}$ and $26 \mathrm{G}$ needles, suspended in DMEM supplemented with $20 \% \mathrm{FBS}$, and cultured in T75 flasks (Falcon). After 3 days, medium was changed and cells were maintained in DMEM with 10\% FBS. The identification of the cells was confirmed by immunofluorescence analysis with an astrocytic marker anti glial fibrillary acidic protein (GFAP) antibodies. Cultures usually contained over 95\% GFAP-positive cells. Infection was performed by 1 hour absorption of $1 \mathrm{pfu} / \mathrm{cell}$ of MHV at 10-14 days post plating.

\subsection{Detection of IFNs by RT-PCR and Southern Blot Analysis}

Total cellular RNA was isolated by the TRIzol Reagent (BRL) by following the manufacturer's protocol. Astrocytes were lysed directly in a culture dish by adding TRIzol Reagent $\left(1 \mathrm{ml}\right.$ per $\left.10 \mathrm{~cm}^{2}\right)$, and passing the cell lysate through a pipette. Tissue samples were homogenized by mechanical disruption between two glass slides, then put in $1 \mathrm{ml}$ of TRIzol Reagent per 50-100 mg of tissue. Amplification grade DNAse I (BRL) was used for digestion of DNA before the RT-PCR reaction.

For RT-PCR reaction, $1 \mu \mathrm{g}$ of RNA was reverse transcribed using a random hexamer primer and SuperScript II reverse transcriptase (BRL), according to the manufacturer's protocol. Reagents of SuperScript Preamplification System for First Strand cDNA Synthesis $\mathrm{Kit}(\mathrm{BRL})$ were used in the PCR. The $\beta$-actin and IFNs primers were purchased from ClonTech. The expected size of $\beta$-actin, IFN- $\alpha$, IFN- $\beta$ and IFN- $\gamma$ are $540 \mathrm{bp}, 294 \mathrm{bp}$, $509 \mathrm{bp}$ and $365 \mathrm{bp}$, respectively. The PCR products were further transblotted to the positive nylon membrane (BMB) in 20xSSC overnight at room temperature. Specific anti-sense probes (obtained from ClonTech or designed by MacVECTOR computer program) for $\beta$ actin and IFNs were labeled with Digoxigenin-11-ddUTP by using Genius 5 Oligonucleotide 3'-End Labeling kit (BMB). After 2 hours prehybridization in $65^{\circ} \mathrm{C}$, the blot was sealed in a hybridization bag with $10 \mathrm{pmol} / \mathrm{ml}$ probe in hybridization solution. The bags were kept in a $65^{\circ} \mathrm{C}$ overnight. After post-hybridization wash, the membrane was blocked 
in blocking solution (BMB) for $30 \mathrm{~min}$, then incubated with sheep anti-digoxigenin antibody (BMB), at a 1:10,000 dilution for $30 \mathrm{~min}$. Detection of signal was done by applying chemiluminescent substrate Lumi-Phos 530 (BMB). The membrane was then exposed to a Kodak x-ray film.

Primers and probes for the RT-PCR reactions and Southern blot analysis

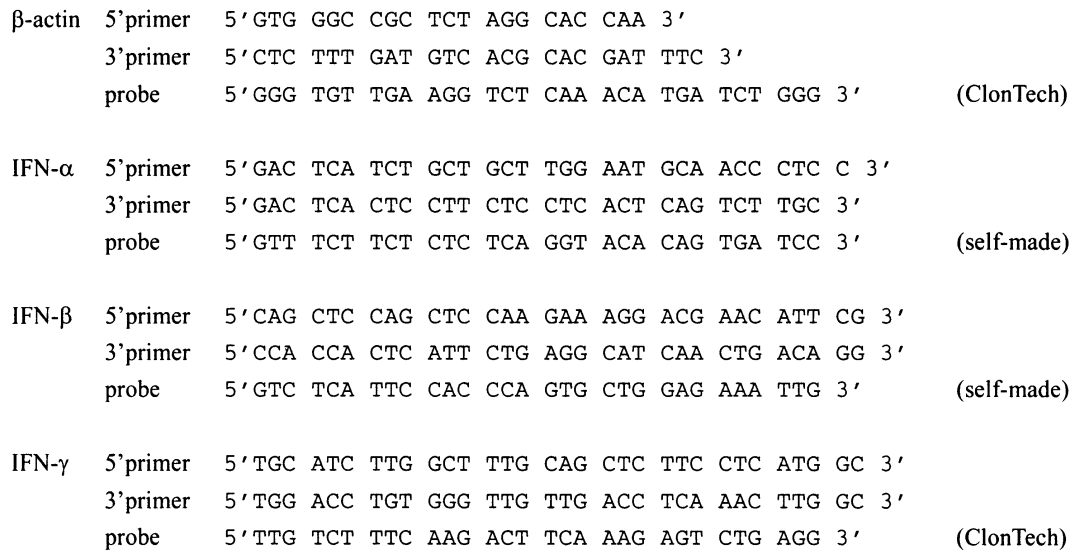

\section{RESULTS}

Uninfected and mock infected cultures did not express any of the three IFNs. Cultures always expressed detectable amounts of $\beta$-actin RNA. Following infection with MHV-A59 or MHV- 2 cultures expressed detectable amounts of IFN- $\beta$ RNA but not IFN- $\alpha$ or IFN- $\gamma$ in addition to $\beta$-actin RNA. The PCR amplified reverse transcribed cDNA product was of the expected size. Southern blot analysis confirmed the specificity of the amplified PCR products showing positive hybridization only with the IFN- $\beta$ and $\beta$-actin probes. PCR analysis at both 1 and 4 days post infection gave identical results.

\section{DISCUSSION}

The experiments shown here provide additional evidence for the role of astrocytes as a local immune cell in the CNS. Astrocytes are capable of producing IFN- $\beta$ following viral infection when exposed to Newcastle Disease virus (NDV) (Lieberman et al., 1989). We now provide evidence that IFN- $\beta$ can also be produced in cultured astrocytes after infection with the demyelinating coronavirus MHV. Although all three interferons RNAs are induced in response to MHV infection in the mouse brain (Lavi and Wang, 1995), upregulation of IFN- $\beta$ RNA in the brain may be due to a local effect of the virus on CNS astrocytes. The other two interferons may be contributed by the inflammatory cells invading the brain during MHV infection. The exact role of IFN-beta in demyelination is not clear. However, the fact that both demyelinating and non-demyelinating strains of MHV produced the same effect of interferon induction in astrocytes may suggest that demyelination is not a function of interferon induction. Additional factors may be responsible for the inability of MHV-2 to produce demyelination. 


\section{ACKNOWLEDGMENTS}

This work was supported by a grant from the National Multiple Sclerosis Society RG-26151/2.

\section{REFERENCES}

Lavi, E., Gilden, D. H., Highkin, M. K., and Weiss, S. R., 1984a, Persistence of MHV-A59 RNA in a slow virus demyelinating infection in mice as detected by in situ hybridization, J. Virol. 51: 563-566.

Lavi, E., Gilden, D. H., Wroblewska, Z., Rorke, L. B., and Weiss, S. R., 1984b, Experimental demyelination produced by the A59 strain of mouse hepatitis virus, Neurology 34: 597-603.

Lavi, E., Suzumura, A., Hirayama, M., Highkin, M. K., Dambach, D. M., Silberberg, D. H., and Weiss, S. R., 1987a, Coronavirus MHV-A59 causes a persistent, productive infection in glial cells, Microbial Pathogenesis 3: 79-86.

Lavi, E., Suzumura, A., Lampson, L. A., Siegel, R. M., Murasko, D. M., Silberberg, D. H., and Weiss, S. R., $1987 \mathrm{~b}$, Expression of MHC class I genes in mouse hepatitis virus (MHV-A59) infection and in multiple sclerosis, Adv. Exp. Med. Biol. 218: 219-222.

Lavi, E., Suzumura, A., Murray, E. M., Silberberg, D. H., and Weiss, S. R., 1989, Induction of MHC class I antigens on glial cells is dependent on persistent mouse hepatitis virus infection, J. Neuroimmunol. 22: $107-111$.

Lavi, E., and Wang, Q., 1995, The protective role of cytotoxic T cells and interferon against coronavirus invasion of the brain, Adv. Exp. Med. Biol. 380: 145-149.

Lavi, E., and Weiss, S. R., 1989, Coronaviruses, in "Clinical and molecular aspects of neurotropic viral infections" (D. H. Gilden, and H. L. Lipton, Eds.), pp. 101-139. Kluwer, Academic Publishers, Boston.

Lieberman, A. P., Pitha, P. M., Shin, H. S., and Shin, M. L., 1989, Production of tumor necrosis factor and other cytokines by astrocytes stimulated with lipopolysaccharide or a neurotropic virus, Proc. Natl. Acad. Sci. USA 86: 6348-6352.

McCarthy, K. D., and de Vellis, J., 1980, Preparation of separate astroglial and oligodendroglial cell cultures from rat cerebral tissue, J. Cell Biol. 85: 890-902.

Suzumura, A., Lavi, E., Bhat, S., Murasko, D. M., Weiss, S. R., and Silberberg, D. H., 1988, Induction of glial cell $\mathrm{MHC}$ antigen expression in neurotropic coronavirus infection: characterization of the $\mathrm{H}-2$ inducing soluble factor elaborated by infected brain cells, J. Immunol., 2068-2072.

Suzumura, A., Lavi, E., Weiss, S. R., and Silberberg, D. H., 1986, Coronavirus infection induces H-2 antigen expression on oligodendrocytes and astrocytes, Science 232: 991-993.

Wang, F. I., Stohlman, S.A., and Fleming, J. O., 1990, Demyelination induced by murine hepatitis virus JHM strain (MHV-4) is immunologically mediated, J. Neuroimmunol. 30: 31-41. 